\title{
Conocimientos, actitudes y prácticas asociados a diarrea aguda en la zona norte de Bucaramanga. Estudio observacional analítico, 2014 - 2015
}

\author{
Pilar Abreu P* \\ Miguel E. Ochoa $V^{* *}$ \\ Hendrik A. Baracaldo C ${ }^{* * *}$ \\ Martha I. Robles $C^{* * * *}$ \\ Angie S. Naranjo $G^{* * * * *}$
}

\begin{abstract}
*Enfermera. Especialista en Administración de Servicios de Salud. Magíster en Educación. Miembro de Grupo de Investigación CARING. Docente Facultad de Ciencias de la Salud. Programa de Enfermería. Universidad Autónoma de Bucaramanga. Bucaramanga. Colombia.

**Médico. Magister en Epidemiología clínica. Observatorio de Salud Pública de Santander. Fundación Oftalmológica de Santander - FOSCAL. Miembro del Grupo de investigaciones Clínicas. Docente Facultad de Ciencias de la Salud. Universidad Autónoma de Bucaramanga. Bucaramanga. Colombia.

***Enfermero. Coordinador Hospitalización Fundación Oftalmológica de Santander - FOSCAL. Miembro de Grupo de Investigación CARING. Docente Facultad de Ciencias de la Salud. Programa de Enfermería. Universidad Autónoma de Bucaramanga. Bucaramanga. Colombia.

****Enfermera. Especialista en Cuidado Crítico con Énfasis en Neonatología. Magíster (C) en Salud Sexual y Reproductiva. Miembro de Grupo de Investigación CARING. Docente Facultad de Ciencias de la Salud. Programa de Enfermería. Universidad Autónoma de Bucaramanga. Bucaramanga. Colombia.

*****Estudiante de Enfermería. Programa de Enfermería. Universidad Autónoma de Bucaramanga. Bucaramanga. Colombia.

Correspondencia: Sra. Pilar Abreu. P. Dirección: Calle 157 № 19-55, Cañaveral Parque. Bucaramanga. Colombia. Teléfono: 3124541545. Correo electrónico: pabreu3@unab.edu.co.
\end{abstract}

\section{RESUMEN}

Introducción: la diarrea aguda y la mortalidad por diarrea continúan siendo un problema de salud pública a nivel mundial, especialmente en los países de ingreso medio o bajo. Gran parte de las enfermedades diarreicas son prevenibles mediante el acceso al agua potable y a servicios adecuados de saneamiento e higiene. Objetivo: caracterizar los conocimientos, actitudes y prácticas de cuidadores de niños menores de cinco años asociados a prevalencia de diarrea. Materiales y métodos: estudio observacional analítico de corte transversal, realizado durante el periodo 2014-2015. Se realizó un muestreo por conglomerados en la comuna norte de la ciudad de Bucaramanga y se aplicó una encuesta dirigida. Resultados: solo uno de cada diez cuidadores conoce todas las características de la diarrea aguda (cambios en la consistencia, número y duración de los síntomas) y ninguno reconoce totalmente los signos de deshidratación. El uso de remedios caseros y la creencia en causas sobrenaturales y esotéricas como causas de diarrea tuvo una alta prevalencia. La menor edad del cuidador, tener aseguramiento y mayor nivel conocimientos sobre la diarrea se asociaron a un mayor reporte de episodios de diarrea en los últimos seis meses. Conclusiones: el $49 \%$ de todos los cuidadores encuestados manifestaron que sus niños han tenido al menos un episodio de diarrea en los últimos seis meses. El nivel de conocimientos de los cuidadores es bajo. Se encontró asociación estadísticamente significativa entre la edad del cuidador, conocimiento de los síntomas diarreicos, régimen de aseguramiento y prevalencia de diarrea en menores de cinco años. MÉD.UIS. 2017;30(1):21-33.

Palabras Clave: Diarrea. Cuidado del Niño. Cuidadores. Conocimientos, Actitudes y Práctica en Salud.

\section{Knowledge, attitudes and practices associated with acute diarrhea in the northern zone of Bucaramanga. An observational analytic study. 2014 to 2015}

\section{ABSTRACT}

Introduction: acute diarrhea and mortality from diarrhea remains a public health problem worldwide, particularly in middle-income countries or low. Much of diarrheal diseases are preventable through access to safe water and adequate sanitation and hygiene. Objective: to characterize the knowledge, attitudes and practices of caregivers of children under five years associated with prevalence of diarrhea. Materials and methods: cross-sectional observational study, made during the period 2014-2015. Cluster sampling was conducted in the northern district of the city of Bucaramanga. A targeted survey was conducted. Results: only one in ten caregivers 
know all the characteristics (changes in consistency, number and duration of symptoms) of acute diarrhea. None fully recognize the signs of dehydration. The use of home remedies and belief in supernatural and esoteric causes as causes of diarrhea had a high prevalence. Caregiver younger, having higher-level assurance and knowledge on diarrhea associated with increased reporting of episodes of diarrhea in the last six months. Conclusions: $49 \%$ of all respondents report that their children caregivers have had at least one episode of diarrhea in the last six months. The level of knowledge of caregivers is low. Age of the caregiver, knowledge about diarrhea symptoms and healthcare affiliation was statistically associated with the onset of diarrhea in children up to five years. MÉD.UIS. 2017;30(1):21-33.

Keywords: Diarrhea. Child Care. Caregivers. Health Knowledge, Attitudes, Practice.

¿Cómo citar este artículo?: Abreu P, Ochoa ME, Baracaldo HA, Robles MI, Naranjo AS. Conocimientos, actitudes y prácticas asociados a diarrea aguda en la zona norte de Bucaramanga. Estudio observacional analítico aplicado durante el periodo 2014-2015. MÉD.UIS. 2017;30(1):21-33.

\section{INTRODUCCIÓN}

Según la Organización Mundial de la Salud (OMS) y la Organización Panamericana de la Salud (OPS) se define como diarrea la aparición de tres o más deposiciones de heces sueltas o líquidas en 24 horas o con una frecuencia mayor que la normal para la persona, acompañada o no de sangre'. En el mundo anualmente se presentan 1700 millones de casos de Enfermedad Diarreica Aguda (EDA), siendo los niños menores de cinco años quienes presentan tres episodios diarreicos al año².

Con respecto a su etiología, el rotavirus es la principal causa de diarrea y está presente en el $40 \%$ de las hospitalizaciones en niños menores de cinco años ${ }^{3}$. Además es el causante del $28 \%$ de la diarrea severa, que resulta en casi diez millones de episodios severos y más de 193000 muertes a escala global4; adicionalmente se reporta que el $72 \%$ de las muertes asociadas a diarrea ocurren antes de los dos años de edad. La malnutrición y la inmunosupresión son factores asociados a mayor riesgo de enfermedades diarreicas potencialmente mortales, es así como ocho de cada diez muertes por diarrea están asociadas a un inadecuado saneamiento del agua e inapropiadas prácticas higiénicas ${ }^{2,5}$.

La EDA tiene como principales complicaciones la deshidratación y desnutrición que pueden llevar a estados de choque, enfermedad renal, intestinal y la muerte. A nivel mundial la EDA es la segunda mayor causa de muerte en niños menores de cinco años y la mortalidad anual estimada es de 760000 niños $^{6}$. En América Latina se calcula que alrededor de 77600 niños menores de cinco años mueren anualmente por enfermedad diarreica debida al consumo de agua no apta, alimentos contaminados y malos hábitos de higiene. En Colombia, en la década 1993 y 2003 se registraron cinco millones de casos de diarrea por causa hídrica con una tasa de mortalidad igual a 5,6 por cada 100 mil niños ${ }^{7}$. Adicionalmente, el Sistema de Vigilancia en Salud Pública (SIVIGILA) en la semana epidemiológica 52 del año 2010 reportó 158 casos de muerte en niños menores de cinco años por EDA.

Además para el año 2014 la tasa de mortalidad por EDA en niños menores de cinco años fue de 3,11 por 100000 habitantes $^{8}$, encontrándose que las poblaciones más afectadas eran las de menores ingresos ${ }^{9}$. Con respecto a su incidencia en Santander, la EDA origina el ocho por ciento de las consultas de urgencias en niños y el siete por ciento se presentan en niños menores de cinco años ${ }^{10}$.

El rol que desempeñan los padres y la familia en la salud del niño es de gran importancia no solamente por tener a su cargo el cuidado y atención del niño en el hogar, sino por definir cuándo el niño está enfermo y qué actitud deben asumir frente a la enfermedad"; así mismo, determina la identificación de signos de alarma, tiempo de consulta en los servicios sanitarios y delimita actividades de prevención, cuidado y tratamiento ante dicha enfermedad. Este comportamiento está dado por componentes globales influyentes en el cuidado, como el punto de vista del mundo, estructura social, lenguaje, cuidados genéricos y contexto ambiental ${ }^{12}$.

Al abordar la cultura y su papel en la noción de salud y enfermedad, es relevante conocer la realidad actual en la percepción y manejo de enfermedades que han prevalecido durante años en la infancia, en este caso la EDA. Actualmente la sociedad presenta gran diversidad de creencias y prácticas que definen y limitan su actuar ante la aparición de una enfermedad, en especial cuando se trata del cuidado de niños. La familia, en especial los padres, continúan simbolizando el núcleo de soporte necesario al implementar actividades de preservación del patrón 
cultural, construcción de hábitos y el desarrollo de actividades dirigidas a la conservación de la salud. Por lo anterior, se busca conocer aspectos importantes que brinden información integral que a futuro se aprovechen para promover en la sociedad hábitos y estilos de vida saludables coherentes con su patrón cultural y autonomía ${ }^{13}$. Es por ello que el objetivo de este estudio es describir los conocimientos, actitudes y prácticas de los cuidadores de niños menores de cinco años sobre EDA en hogares de la zona de norte de Bucaramanga y compararlos con la prevalencia de episodios de EDA.

\section{Materiales y Métodos}

\section{DISEÑO DE ESTUDIO}

Corresponde a un estudio observacional analítico de corte transversal realizado en el periodo 2014-2015 con cuidadores de niños menores de cinco años en la zona norte de Bucaramanga comuna uno, quienes residían en la localidad al menos los últimos seis meses antes de la aplicación de una encuesta dirigida y decidieron participar anónima y voluntariamente.

Se realizó la búsqueda de experiencias previas relacionadas con el diseño de instrumentos que permitan la recolección de datos sobre la percepción de los cuidadores respecto a los signos y síntomas de la EDA y la importancia que tiene consultar de manera prioritaria a los servicios de salud y se logró identificar en la estrategia de Atención Integral de las Enfermedades Prevalentes de la Infancia (AIEPI) indicaciones generales para el reconocimiento y tratamiento del cuadro clínico del niño, además de una ficha de entrevista familiar domiciliaria que indaga sobre factores de riesgo y que permite la interrelación con el objetivo general y específicos del proyecto investigativo.

Con referencia al instrumento, correspondió a una ficha de entrevista a la familia en el domicilio. El grupo de investigación inicia la adaptación del documento primario aportado en el instructivo Operaciones Investigativas Sobre Enfermedades Prevalentes de la Infancia AIEPI-OMS 2001. Así mismo, se tuvieron en cuenta las discusiones y recomendaciones de artículos escogidos a partir de la revisión bibliográfica para realizar la adaptación del instrumento.

En la selección del personal encuestador se tuvieron en cuenta los siguientes criterios de selección: a) estudiantes del programa de enfermería Universidad
Autónoma de Bucaramanga (UNAB), b) estar o haber cursado $\mathrm{V}$ nivel correspondiente al área de Cuidado Holístico al Niño y Adolescente, c) evidenciar interés en la participación del proyecto; posterior a su aplicación, tres estudiantes fueron seleccionados. Despues de ello, se realizó una prueba piloto con el propósito de comprobar y hacer ajustes al instrumento. El diseño metodológico de esta se planteó en dos etapas. La primera etapa correspondió al desarrollo de la prueba inicial denominada "LIVE", etapa de desarrollo y la segunda etapa de cierre denominada "CLOSE", etapa de análisis. Fases interconectadas que buscaron evaluar la idoneidad del instrumento, calcular el tiempo necesario para desarrollar completamente la entrevista y determinar la calidad de la tarea del encuestador.

Las encuestas se aplicaron en los meses de febrero a noviembre de 2014, estas contienen información organizada en seis apartados a saber: datos demográficos del niño, composición familiar, características de la vivienda y conocimientos del cuidador respecto a etiología, signos de alarma, tratamiento de la diarrea y actitudes y prácticas en diarrea. El muestreo realizado fue probabilístico y multietápico, en el cual las unidades primarias fueron los barrios, las secundarias fueron las manzanas; pero no se contó con una cartografía actualizada ni detallada para una optimización de los conglomerados y segmentos, por lo cual se usó una cartografía satelital de acceso libre. Se escogieron las viviendas de las unidades secundarias hasta completar el tamaño calculado para cada segmento, en una zona que incluye viviendas de estrato bajo y medio-bajo ${ }^{9}$ de la ciudad de Bucaramanga (Comuna Norte).

Los datos de los instrumentos fueron codificados y digitados por duplicado por personal del Observatorio de Salud Pública de Santander (OSPS), en hojas electrónicas de Excel, y las bases fueron posteriormente depuradas y validadas electrónicamente y analizadas utilizando el software STATA. No se registró identidad ni códigos ligantes a los domicilios de los cuidadores o niños.

\section{CARACTERIZACIÓN DE LA POBLACIÓN}

Se calculó un tamaño de muestra de 318 familias para un nivel de confianza de $95 \%$ y un poder de $80 \%$, con prevalencia del factor de riesgo de $33 \%$ y una diferencia de proporciones de 16\%. Calculada para una hipótesis bilateral, basada en los datos de motivo de 
consulta con diagnóstico de diarrea y gastroenteritis de presunto origen infeccioso (CIE 100 - Aog) E.S.E ISABU en el área Norte de Bucaramanga entre Enero - Septiembre 2012. Al revisar la información recolectada se encontraron seis encuestas de familias con niños menores de seis meses, por lo tanto se redujo la muestra a 313 familias.

Dentro de los criterios de inclusión se definieron para selección cuidadores de niños entre los seis meses y cinco años de edad, que fueran residentes de la zona norte de Bucaramanga, comuna uno, al menos en los últimos seis meses antes de la aplicación del instrumento y que decidieran participar voluntariamente. Se excluyeron los cuidadores que no tuvieran al menos un niño mayor de seis meses, puesto que la prevalencia indagada es para el periodo de los últimos seis meses.

Dentro de las estrategias utilizadas para evitar el sesgo en la información recolectada se tuvo en cuenta: garantizar que la persona que suministraba la información fuera el real cuidador del niño y la reformulación de las preguntas en forma más específica y sencilla al notar dificultad de entendimiento por parte del entrevistado.

\section{Definición de VARIABLES}

Se recolectaron variables de naturaleza numérica (número de familias en el hogar, número de niños menores de cinco años, edad del niño y cuidadores, número de personas que conviven con el niño, grifos, número de síntomas y signos de alarma identificados por el cuidador, número de episodios de diarrea en los últimos seis meses) y variables cualitativas (actitudes, conocimientos y prácticas caseras) tanto ordinales como nominales. Las variables se resumieron en promedios o porcentajes y de manera análoga como gráficos según su naturaleza. Se realizaron análisis bivariados y estratificados previos (no incluidos) para escoger las variables candidatas a un modelo multivariado de regresión binomial según criterios de modelamiento ${ }^{9}$, y presentar las Razones de Prevalencia (RP) ajustadas, con sus respectivos intervalos de confianza al 95\% con nivel de significancia $a=0,05$.

Factores sociodemográficos, circunstancias materiales y de vivienda: número de personas en el hogar, número de niños menores de cinco años, estrato socioeconómico, régimen de seguridad social, disponibilidad de acueducto, alcantarillado, periodicidad de recolección de basuras, numero de grifos en la vivienda, fuente principal de agua para aseo y para preparación de alimentos, presencia de mascotas o animales de corral, tipo de residencia (urbana/rural, propia/arrendada).

Características del cuidador: parentesco, edad, nivel educativo.

Conocimientos del cuidador sobre definición, síntomas, etiología, signos de alarma diarrea, tratamiento y prevención: definición de la diarrea, episodios de diarrea presentados en los últimos seis meses, causas de la diarrea, signos de alarma para acudir a los servicios de salud, signos de deshidratación, tratamiento de la diarrea, conocimiento de las Sales de Rehidratación Oral (SRO), uso de SRO, preparación de SRO-, uso de fórmulas comerciales de sales de rehidratación oral, relación de la cantidad de líquidos y alimentos que se le proporcionan al niño cuando tiene diarrea, cuidados brindados por el cuidador cuando el niño tiene diarrea preparados caseros más utilizados.

Actitudes y prácticas frente a episodios de diarrea aguda: ¿qué hace el cuidador cuando el niño tiene diarrea?, ¿qué cree que es la diarrea?, ¿cuál es la causa de la diarrea?, prácticas que evitan la aparición de la diarrea, ¿dónde se adquieren los conocimientos sobre la diarrea?

\section{Resultados}

\section{CARACTERÍSTICAS DEL CUIDADOR}

Se encuestaron 313 cuidadores (tres hombres, 310 mujeres) de niños entre seis meses y cinco años de 12 barrios de la comuna 1 , con un promedio de edad de $32 \pm 12$ años. Del total de cuidadores cinco (1,59\%) eran menores de edad y el $45 \%$ se encontraba entre los 20 y los 39 años. El cuidador más joven tenía 16 años al momento de la encuesta y el mayor, 76 años. Aproximadamente el diez por ciento de los cuidadores no habían completado la educación primaria, y el 44\% contaban con educación básica secundaria y media (Ver tabla 1). 
ENERO - ABRIL

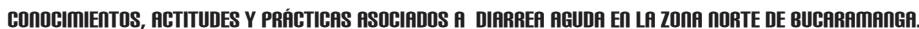
ESTUDIO OBSERVACIONAL AกALITICO APLICADO DURARTE EL PERIODO 2014-2015

Tabla 1. Características del cuidador en la comuna norte de Bucaramanga.

\begin{tabular}{|c|c|c|}
\hline Cuidador principal del niño & $\mathbf{n}$ & Porcentaje \\
\hline Madre & 245 & $78,28 \%$ \\
\hline Abuela & 53 & $16,93 \%$ \\
\hline Tía & 6 & $1,92 \%$ \\
\hline Padre & 3 & $0,96 \%$ \\
\hline Bisabuela & 2 & $0,64 \%$ \\
\hline Hermana & 2 & $0,64 \%$ \\
\hline Cuidadora & 1 & $0,32 \%$ \\
\hline Madrina & 1 & $0,32 \%$ \\
\hline Total & 313 & $100 \%$ \\
\hline $\begin{array}{l}\text { Nivel educativo del } \\
\text { cuidador }\end{array}$ & & \\
\hline Secundaria completa & 111 & $35,46 \%$ \\
\hline Secundaria incompleta & 83 & $26,52 \%$ \\
\hline Primaria completa & 55 & $17,57 \%$ \\
\hline Primaria incompleta & 28 & $8,95 \%$ \\
\hline Técnico completo & 23 & $7,35 \%$ \\
\hline Técnico incompleto & 9 & $2,88 \%$ \\
\hline Analfabeta & 1 & $0,32 \%$ \\
\hline No estudió pero lee & 2 & $0,64 \%$ \\
\hline No lee pero sabe contar & 1 & $0,32 \%$ \\
\hline Total & 313 & $100 \%$ \\
\hline $\begin{array}{l}\text { Edad promedio del cuidador } \\
\pm \text { DS }\end{array}$ & \multicolumn{2}{|c|}{$31,71 \pm 12,52$} \\
\hline
\end{tabular}

Fuente: autores

\section{FACTORES SOCIODEMOGRÁFICOS CIRCUNSTANCIAS MATERIALES Y DE VIVIENDA}

Las familias estuvieron conformadas por tres a 14 personas, con una media de 6,4 \pm 2 personas por hogar, donde el $52 \%$ de los niños eran mujeres. La mayoría pertenecía al régimen contributivo y subsidiado. Las viviendas eran principalmente estrato $3(32 \%)$ y dos(62\%). Solo cuatro viviendas carecían de servicio de acueducto y seis no contaban con alcantarillado. Había en promedio tres grifos por vivienda y el $89 \%$ de las familias utilizan el lavaplatos para obtener el agua para la preparación de alimentos, llama la atención que el lavadero es la fuente de aseo personal más frecuente. La recolección de basuras es semanal en todos los sectores. El 45\% de los hogares tenían al menos una mascota. Solo hubo animales de corral en una vivienda (conejos). Las características sociodemográficas y circunstancias materiales se resumen en la tabla 2 (Ver Tabla 2)
CONOCIMIENTOS DEL CUIDADOR SOBRE DEFINICIÓN, SÍNTOMAS, ETIOLOGÍA, (SIGNOS DE ALARMA DE LA DIARREA), TRATAMIENTO Y PREVENCIÓN

Respecto al conocimiento sobre las características de la diarrea solo el $4 \%$ identificó correctamente todos los signos y síntomas (cambios en la consistencia, aumento en el número y duración de los síntomas). El $93,5 \%$ de los cuidadores ignoraban la duración mínima de los síntomas gastrointestinales requerida para que se considere que corresponden a un episodio de EDA. Los cambios en la consistencia y la frecuencia de la deposición fueron ignorados por el $26 \%$ y el $30 \%$ de los cuidadores, respectivamente.

Las causas de diarrea fueron reconocidas por menos de la mitad de los encuestados, siendo la ingesta de alimentos contaminados (47,8\%) y el agua (40\%) la respuesta de mayor frecuencia. No obstante, otras creencias como la "descuajadura", el "mal de ojo" y la brujería fueron reportadas por la tercera parte de los cuidadores (30,48\%), (Ver Figura 1).

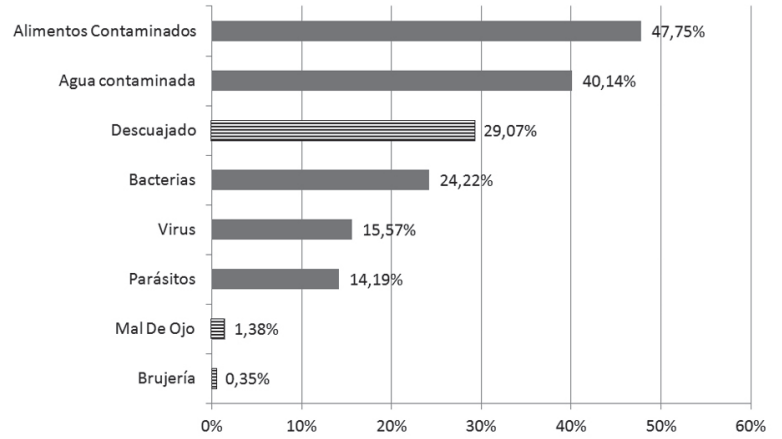

Figura 1. Causas de diarrea reportadas por los cuidadores.

Fuente: autores

Los signos de deshidratación más frecuentemente reconocidos no superan el $40 \%$ de los encuestados. Solo el $37 \%$ de los cuidadores reconoce como signos de deshidratación la sequedad en mucosa oral y el hundimiento de ojos y mejillas. El reconocimiento de los demás signos estuvo presente en menos del $20 \%$ de los cuidadores. El llanto sin lágrimas y la disminución de la diuresis fueron reconocidos por menos del cuatro por ciento de los cuidadores. Los signos de alarma para consulta inmediata reportados en mayor porcentaje fueron la duración de la diarrea por más de 24 horas y la fiebre de más de 39 grados centígrados. Sin embargo, el $35 \%$ de los cuidadores no reconocía la duración de la diarrea como signo de alarma y el $69 \%$ no reconoció la fiebre como criterio de consulta. La intolerancia a la vía oral, la deshidratación y las heces con sangre o pus, fueron identificadas por menos del $15 \%$ de los cuidadores. 
Tabla 2. Características sociodemográficas y circunstancias materiales y de vivienda en los hogares de la comuna norte de Bucaramanga

\begin{tabular}{|c|c|c|}
\hline $\begin{array}{c}\text { Régimen de } \\
\text { seguridad social }\end{array}$ & $\mathbf{n}$ & Porcentaje \\
\hline Contributivo & 174 & $55,60 \%$ \\
\hline Subsidiado & 120 & $38,34 \%$ \\
\hline $\begin{array}{l}\text { Especial o } \\
\text { Excepción }\end{array}$ & 10 & $3,19 \%$ \\
\hline No Asegurado & 8 & $2,56 \%$ \\
\hline Prepagada & 1 & $0,32 \%$ \\
\hline Total & 313 & $100 \%$ \\
\hline \multicolumn{3}{|l|}{ Tipo de vivienda } \\
\hline Casa & 214 & $68,37 \%$ \\
\hline Apartamento & 67 & $21,41 \%$ \\
\hline Tugurio & 27 & $8,63 \%$ \\
\hline Pieza & 5 & $1,60 \%$ \\
\hline Total & 313 & $100 \%$ \\
\hline \multicolumn{3}{|l|}{ Estrato } \\
\hline 1 & 195 & $62,30 \%$ \\
\hline 2 & 103 & $32,91 \%$ \\
\hline 3 & 15 & $4,79 \%$ \\
\hline Total & 313 & $100 \%$ \\
\hline \multicolumn{3}{|l|}{$\begin{array}{c}\text { Área De } \\
\text { Residencia* }\end{array}$} \\
\hline Rural & 4 & $1,28 \%$ \\
\hline Urbana & 307 & $98,08 \%$ \\
\hline Total & 311 & $99,36 \%$ \\
\hline \multicolumn{3}{|l|}{$\begin{array}{c}\text { ¿La vivienda es } \\
\text { propia? }\end{array}$} \\
\hline No & 174 & $55,59 \%$ \\
\hline Sí & 139 & $44,41 \%$ \\
\hline Total & 313 & $100 \%$ \\
\hline \multicolumn{3}{|l|}{$\begin{array}{c}\text { ¿Hay animales en } \\
\text { la casa? }\end{array}$} \\
\hline No & 172 & $55 \%$ \\
\hline Sí & 141 & $45 \%$ \\
\hline Total & 313 & $100 \%$ \\
\hline \multicolumn{3}{|l|}{$\begin{array}{c}\text { Servicio de } \\
\text { alcantarillado }\end{array}$} \\
\hline No & 6 & $1,92 \%$ \\
\hline Sí & 307 & $98,08 \%$ \\
\hline Total & 313 & $100 \%$ \\
\hline Agua - higiene & & \\
\hline
\end{tabular}

\begin{tabular}{|c|c|c|}
\hline $\begin{array}{c}\text { Régimen de } \\
\text { seguridad social }\end{array}$ & $\mathbf{n}$ & Porcentaje \\
\hline Lavadero & 137 & $43,77 \%$ \\
\hline Lavamanos & 109 & $34,82 \%$ \\
\hline Lavaplatos & 27 & $8,63 \%$ \\
\hline $\begin{array}{l}\text { Otras dentro de la } \\
\text { casa }\end{array}$ & 11 & $3,51 \%$ \\
\hline Llave o Grifo & 8 & $2,56 \%$ \\
\hline Regadera & 5 & $1,60 \%$ \\
\hline Pozo, Tanque & 4 & $1,28 \%$ \\
\hline $\begin{array}{l}\text { Otras Fuera de la } \\
\text { casa }\end{array}$ & 4 & $1,28 \%$ \\
\hline Otro Lugar & 3 & $0,96 \%$ \\
\hline $\begin{array}{l}\text { Lavamanos, } \\
\text { Lavadero }\end{array}$ & 2 & $0,64 \%$ \\
\hline Otra Fuente & 1 & $0,32 \%$ \\
\hline Grifo del baño & 1 & $0,32 \%$ \\
\hline Ducha & 1 & $0,32 \%$ \\
\hline Total & 313 & $100 \%$ \\
\hline \multicolumn{3}{|l|}{$\begin{array}{l}\text { Lugar de donde } \\
\text { se obtiene el agua } \\
\text { para los alimentos }\end{array}$} \\
\hline Lavaplatos & 280 & $89,46 \%$ \\
\hline Lavadero & 16 & $5,11 \%$ \\
\hline Lavamanos & 5 & $1,60 \%$ \\
\hline $\begin{array}{l}\text { Otras fuera de la } \\
\text { casa }\end{array}$ & 5 & $1,60 \%$ \\
\hline Otra llave o grifo & 3 & $0,96 \%$ \\
\hline Otra fuente & 2 & $0,64 \%$ \\
\hline Pozo, tanque & 2 & $0,64 \%$ \\
\hline Total & 313 & $100 \%$ \\
\hline \multicolumn{3}{|l|}{$\begin{array}{l}\text { Periodicidad de } \\
\text { recolección de } \\
\text { basuras }\end{array}$} \\
\hline Semanal & 313 & $100,00 \%$ \\
\hline $\begin{array}{l}\text { Promedio personas } \\
\text { en el hogar } \pm \text { DS }\end{array}$ & $5,41 \pm 1,93$ & \\
\hline $\begin{array}{l}\text { Promedio niños } \\
\text { menores de } 5 \text { años } \\
\pm \text { DS }\end{array}$ & $1,13 \pm 1,46$ & \\
\hline $\begin{array}{l}\text { Promedio grifos en } \\
\text { la casa } \pm \text { DS }\end{array}$ & $3,05 \pm 1,03$ & \\
\hline
\end{tabular}

*2 registros sin dato de área de residencia.

Fuente: autores 
Aproximadamente siete de cada diez cuidadores reconoce las SRO como tratamiento para la deshidratación por diarrea aguda, y nueve de cada diez considera que la reposición de líquidos hace parte del manejo de la diarrea. Sin embargo, el 90\% conoce los preparados comerciales de rehidratación aunque algunos incluyen también fórmulas de hidratación deportivas como parte del manejo. De los que reconocen las SRO, menos del $60 \%$ saben prepararlas correctamente.

Como actividades preventivas, se encontró que predomina en los cuidadores hervir el agua con un $57,7 \%$, seguida por el lavado de manos con $45,3 \%$; otra actividad que se resalta es la higiene del hogar con un $34,6 \%$. Se evidenció que el $48,44 \%$ de la población no reporta episodios de diarrea; el $47,75 \%$ reporta menos de tres episodios en este periodo, el 3,8\% reportó más de tres episodios. El $49 \%$ de todos los encuestados manifiestan que sus niños han tenido al menos un episodio de diarrea en los últimos seis meses. En la Tabla 3 se relacionan los conocimientos del cuidador sobre definición, síntomas, etiología, signos de alarma para diarrea, tratamiento y prevención (Ver Tabla 3).

Tabla 3. Conocimientos del cuidador sobre definición, síntomas, etiología, signos de alarma, tratamiento y prevención de la diarrea.

\begin{tabular}{|c|c|c|}
\hline & $\mathbf{n}$ & Porcentaje \\
\hline \multicolumn{3}{|c|}{ DEFINICIÓN DE DIARREA } \\
\hline \multicolumn{3}{|c|}{ Aumento en el número de deposiciones } \\
\hline No & 114 & $36,42 \%$ \\
\hline Sí & 199 & $63,58 \%$ \\
\hline Total & 313 & $100 \%$ \\
\hline \multicolumn{3}{|c|}{ Cambio en la consistencia de las heces } \\
\hline No & 92 & $29,39 \%$ \\
\hline Sí & 221 & $70,61 \%$ \\
\hline Total & 313 & $100 \%$ \\
\hline \multicolumn{3}{|c|}{ ETIOLOGÍA } \\
\hline \multicolumn{3}{|c|}{ Bacterias } \\
\hline No & 237 & $75,72 \%$ \\
\hline Sí & 76 & $24,28 \%$ \\
\hline Total & 313 & $100 \%$ \\
\hline \multicolumn{3}{|c|}{ Virus } \\
\hline No & 266 & $84,98 \%$ \\
\hline Sí & 47 & $15,02 \%$ \\
\hline Total & 313 & $100 \%$ \\
\hline \multicolumn{3}{|c|}{ Parásitos } \\
\hline No & 271 & $86,58 \%$ \\
\hline
\end{tabular}

\begin{tabular}{|c|c|c|}
\hline & $\mathbf{n}$ & Porcentaje \\
\hline Sí & 42 & $13,42 \%$ \\
\hline Total & 313 & $100 \%$ \\
\hline \multicolumn{3}{|c|}{ Agua contaminada } \\
\hline No & 187 & $59,74 \%$ \\
\hline Sí & 126 & $40,26 \%$ \\
\hline Total & 313 & $100 \%$ \\
\hline \multicolumn{3}{|c|}{ Alimentos contaminados } \\
\hline No & 165 & $52,72 \%$ \\
\hline Sí & 148 & $47,28 \%$ \\
\hline Total & 313 & $100 \%$ \\
\hline
\end{tabular}

\begin{tabular}{|l|l|l|}
\hline \multicolumn{3}{|c|}{ Señales de deshidratación } \\
\hline No & 279 & $89,14 \%$ \\
\hline Sí & 34 & $10,86 \%$ \\
\hline Total & 313 & $100 \%$ \\
\hline \multicolumn{3}{|c|}{ Diarrea por más de 24 horas } \\
\hline No & 106 & $33,87 \%$ \\
\hline Sí & 207 & $66,13 \%$ \\
\hline Total & 313 & $100 \%$ \\
\hline
\end{tabular}

\begin{tabular}{|l|l|l|}
\hline \multicolumn{3}{|c|}{ Fiebre de 39 grados o más } \\
\hline No & 213 & $68,05 \%$ \\
\hline Sí & 100 & $31,95 \%$ \\
\hline Total Heces con sangre o pus \\
\hline \multicolumn{3}{|c|}{ Hen } \\
\hline No & 303 & $96,81 \%$ \\
\hline Sí & 10 & $3,19 \%$ \\
\hline Total & 313 & $100 \%$ \\
\hline
\end{tabular}

CUÁLES SON LOS SIGNOS DE DESHIDRATACIÓN

\begin{tabular}{|l|l|l|}
\hline \multicolumn{3}{|c|}{ Boca y lengua resecas } \\
\hline No & 199 & $63,58 \%$ \\
\hline Sí & 114 & $36,42 \%$ \\
\hline Total Llanto sin lágrimas \\
\hline \multicolumn{3}{|c|}{} \\
\hline No & 313 & $100 \%$ \\
\hline Sí & 10 & $96,81 \%$ \\
\hline Total & 313 & $3,19 \%$ \\
\hline \multicolumn{2}{|c|}{ No mojar los pañales por más de 3 horas } \\
\hline No & 312 & $99,68 \%$ \\
\hline Sí & 1 & $0,32 \%$ \\
\hline Total & 313 & $100 \%$ \\
\hline
\end{tabular}




\begin{tabular}{|c|c|c|}
\hline & $\mathbf{n}$ & Porcentaje \\
\hline \multicolumn{3}{|c|}{$\begin{array}{c}\text { Ojos, mejillas o el área del cráneo blanda } \\
\text { hundidos }\end{array}$} \\
\hline No & 197 & $62,94 \%$ \\
\hline Sí & 116 & $37,06 \%$ \\
\hline Total & 313 & $100 \%$ \\
\hline \multicolumn{3}{|c|}{ Tener fiebre alta } \\
\hline No & 216 & $69,10 \%$ \\
\hline Sí & 97 & $30,90 \%$ \\
\hline Total & 313 & $100 \%$ \\
\hline \multicolumn{3}{|c|}{ Llanto sin control o quietud anormal } \\
\hline No & 269 & $85,94 \%$ \\
\hline Sí & 44 & $14,06 \%$ \\
\hline Total & 313 & $100 \%$ \\
\hline \multicolumn{3}{|c|}{ Pliegue cutáneo lento } \\
\hline No & 296 & $94.57 \%$ \\
\hline Sí & 17 & $5,43 \%$ \\
\hline Total & 313 & $100 \%$ \\
\hline \multicolumn{3}{|c|}{ Bebe ávidamente } \\
\hline No & 256 & $81.80 \%$ \\
\hline Sí & 57 & $18,20 \%$ \\
\hline Total & 313 & $100 \%$ \\
\hline \multicolumn{3}{|c|}{ TRATAMIENTO } \\
\hline \multicolumn{3}{|c|}{ Conoce las SRO } \\
\hline No & 98 & $31,30 \%$ \\
\hline Sí & 215 & $68,70 \%$ \\
\hline Total & 313 & $100 \%$ \\
\hline
\end{tabular}

\begin{tabular}{|l|l|l|}
\hline & \multicolumn{1}{|c|}{$\mathbf{n}$} & Porcentaje \\
\hline Sí & 136 & $43,45 \%$ \\
\hline Total Hervir el agua \\
\hline \multicolumn{3}{|c|}{313} \\
\hline No & 135 & 100 \\
\hline Sí & 178 & $53,13 \%$ \\
\hline Total & 313 & $100 \%$ \\
\hline \multicolumn{3}{|c|}{ Evitar contacto con las heces } \\
\hline No & 306 & $97,76 \%$ \\
\hline Sí & 7 & $2,24 \%$ \\
\hline Total & 313 & $100 \%$ \\
\hline
\end{tabular}

LA DIARREA PUEDE SER CAUSADA POR MITOS COMO

\begin{tabular}{|l|l|l|}
\hline \multicolumn{3}{|c|}{ Mal de ojo } \\
\hline No & 309 & $98,72 \%$ \\
\hline Sí & 4 & $1,28 \%$ \\
\hline Total & 313 & $100 \%$ \\
\hline \multicolumn{3}{|c|}{ Descuajado } \\
\hline No & 226 & $72,20 \%$ \\
\hline Sí & 87 & $27,80 \%$ \\
\hline Total & 313 & $100 \%$ \\
\hline \multicolumn{3}{|c|}{ Brujería } \\
\hline No & 312 & $99,68 \%$ \\
\hline Sí & 1 & $0,32 \%$ \\
\hline Total & 313 & $100 \%$ \\
\hline
\end{tabular}

Fuente: autores

\section{ACTITUDES Y PRÁCTICAS FRENTE A EPISODIOS DE DIARREA AGUDA}

\begin{tabular}{|l|l|l|}
\hline No & 167 & $53,35 \%$ \\
\hline Sí & 146 & $46,65 \%$ \\
\hline Total & 313 & $100 \%$ \\
\hline
\end{tabular}

\begin{tabular}{|l|l|l|}
\hline \multicolumn{3}{|c|}{ El agua debe ser potable } \\
\hline No & 165 & $52,72 \%$ \\
\hline Sí & 148 & $47,28 \%$ \\
\hline Total & 313 & $100 \%$ \\
\hline
\end{tabular}

PREVENCIÓN DE LA DIARREA

Se puede prevenir la diarrea

\begin{tabular}{|l|l|l|}
\hline No & 284 & $90,73 \%$ \\
\hline Sí & 29 & $9,27 \%$ \\
\hline Total & 313 & $100 \%$ \\
\hline \multicolumn{3}{|c|}{ ACCIONES PARA PREVENIR LA DIARREA } \\
\hline \multicolumn{3}{|c|}{ Lavado de manos } \\
\hline No & 177 & $56,55 \%$ \\
\hline
\end{tabular}

La creencia en las causas sobrenaturales y las "descuajaduras", ocupó el tercer lugar entre las causas reportadas de diarrea. Aproximadamente el $18 \%$ de los cuidadores prefieren acudir al sobandero o curandero para el tratamiento de la diarrea, por otro lado, el $31 \%$ prefiere tratar a su niño en casa y el $47 \%$ llevarlo a un centro asistencial.

El 64\% de los cuidadores utiliza preparados caseros en el manejo de la EDA entre los cuales los más frecuentes son aquellos que incluyen la guayaba, la manzanilla y el arroz tostado. Con respecto a la prevención de la diarrea, uno de cada diez cuidadores piensa que no puede evitar los episodios de diarrea. Por otro lado los cuidadores obtienen información principalmente de familiares (59,42\%), instituciones de salud (39,62\%) e instituciones educativas (13,74\%), el 3,51\% obtiene 


\section{ENERO - ABRIL}

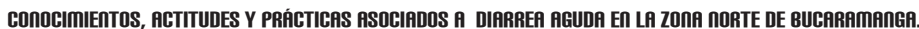
ESTUDIO OBSERVGCIOกAL AกRLITICO APLICADO DURAITE EL PERIODO 2014-2015

información de curanderos y sobanderos. En la Figura 2 se relacionan las principales fuentes de información y sus respectivos porcentajes (Ver Figura 2).

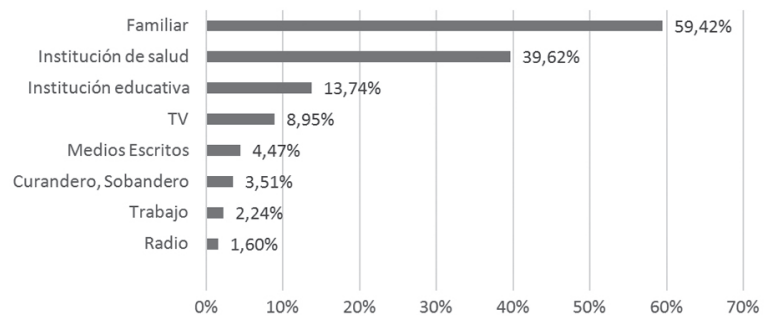

Figura 2. Fuentes de información de los cuidadores sobre la diarrea. * Las respuestas no son mutuamente excluyentes. Puede haber más de una fuente en algunos cuidadores.

Fuente: autores

En relación a prácticas el $86,58 \%$ de los cuidadores aumenta la administración de líquidos y el 49,84\% disminuye la cantidad de sólidos. Con respecto al uso de SRO para el tratamiento de la EDA el 52,08\% no las usa y el $84,66 \%$ hace uso de las fórmulas comerciales ya preparadas (Ver Tabla 4).

ASOCIACIÓN ENTRE LAS CARACTERÍSTICAS DEL CUIDADOR Y LA PRESENCIA DE EPISODIOS DE DIARREA EN LOS ÚLTIMOS SEIS MESES

Se encontró asociación estadísticamente significativa entre los episodios de diarrea y la edad del cuidador, el pertenecer al estrato más alto (estrato 3 ) y los niveles de aseguramiento más bajos (estrato 1 y 2 ) (Ver Tabla 5), en las comparaciones bivariadas. En el modelo multivariado, se encontró que por cada síntoma identificado correctamente, la probabilidad de reporte de diarrea en los últimos seis meses aumenta 1,26 veces. Tener aseguramiento aumenta 1,29 veces la probabilidad de reporte, mientras que la edad del cuidador se asoció inversamente a la probabilidad de reporte de diarrea en los últimos seis meses $(R P=0,99)$. La fuente de conocimientos sobre la diarrea, el uso de preparados caseros, el estrato o la creencia en la descuajadura no mostraron diferencias estadísticamente significativas (Ver Tabla 6).

\section{Discusión}

Gallardo-Lizarazo en el estudio realizado en Bucaramanga encontró que un $43 \%$ de los cuidadores de niños menores de cinco años con EDA tiene estudios de primaria, y el $65 \%$ de estos cuidadores son las madres quienes reconocieron por lo menos tres signos de deshidratación ${ }^{14}$, mientras que en el presente estudio el 35\% de los cuidadores completaron la secundaria, el $78 \%$ son las madres las cuales reconocieron por lo menos un signo de deshidratación lo cual muestra que no hay relación directa con el nivel educativo y los conocimientos relacionados con la enfermedad. Los signos de deshidratación fueron los menos identificados junto con los signos de alarma. Ningún cuidador identifico más de cuatro signos de deshidratación, el $23 \%$ no identificó ninguno de los signos y el $91 \%$ identifico dos o menos signos de deshidratación; datos similares reportan en la investigación de Delgado en el 2006 donde aproximadamente la mitad de la población de madres estudiadas conocía como mínimo dos signos de alarma en EDA ${ }^{15}$.

Se encontró en relación a la definición de diarrea que solo un cuatro por ciento de los cuidadores identifican todos los signos y síntomas correctamente. Del 96\% restante, tres de cada diez no identifica los cambios en la consistencia de la deposición como signo de diarrea y el $40 \%$ no identificó el aumento en la frecuencia de la deposición. Resultados obtenidos por otros autores muestran un mayor reconocimiento de los signos y síntomas por parte de los cuidadores ${ }^{16,17,18}$. Jhonnel y colaboradores, encontraron un $75,6 \%$ de la población estudiada define la diarrea como heces líquidas y aumento del número de deposiciones ${ }^{19}$.

Tabla 4. Manejo de líquidos y alimentación durante los episodios de diarrea reportados por los cuidadores de niños menores de cinco años de la comuna norte de Bucaramanga.

\begin{tabular}{|l|c|c|}
\hline \multicolumn{1}{|c|}{ Cantidad líquidos } & $\mathbf{n}$ & Porcentaje \\
\hline Menor cantidad & 4 & $1,28 \%$ \\
\hline Igual cantidad & 38 & $12,14 \%$ \\
\hline Mayor cantidad & 271 & $86,58 \%$ \\
\hline Total & 313 & 100 \\
\hline \multicolumn{3}{|c|}{ Cantidad sólidos } \\
\hline No ofrece sólidos & 5 & $1,60 \%$ \\
\hline Menor cantidad & 156 & $49,84 \%$ \\
\hline Igual cantidad & 138 & $44,08 \%$ \\
\hline Mayor cantidad & 14 & $4,47 \%$ \\
\hline Total & 313 & $100 \%$ \\
\hline \multicolumn{1}{|c|}{ Otras sales de rehidratación oral (Pedialyte, etc) } \\
\hline No & 48 & $15,34 \%$ \\
\hline Sí & 265 & $84,66 \%$ \\
\hline Total & 313 & $100 \%$ \\
\hline \multicolumn{3}{|c|}{ Ha utilizado sales de rehidratación oral } \\
\hline No & 163 & $52,08 \%$ \\
\hline Sí & 150 & $47,92 \%$ \\
\hline Total & 313 & 100 \\
\hline
\end{tabular}

Fuente: autores 
Tabla 5. Distribución de diarrea aguda según estrato socioeconómico en los diez barrios con más frecuencia de episodios en la comuna norte.

\begin{tabular}{|l|c|c|c|c|c|c|}
\hline \multicolumn{1}{|c|}{ Barrios } & Encuestados & $\begin{array}{c}\text { Estrato } \\
\text { Socioeconómico }\end{array}$ & Sin episodios & Con episodios & \% Sin diarrea & \% Diarrea \\
\hline Cervunión & 23 & 1 & 7 & 16 & $30,43 \%$ & $69,57 \%$ \\
\hline Café Madrid & 29 & 2 & 11 & 18 & $37,93 \%$ & $62,07 \%$ \\
\hline Betania & 15 & 2 & 6 & 9 & $40,00 \%$ & $60,00 \%$ \\
\hline Villa Rosa & 23 & $1-2$ & 11 & 12 & $47,83 \%$ & $52,17 \%$ \\
\hline Alegría 2 & 10 & $1-2$ & 5 & 5 & $50,00 \%$ & $50,00 \%$ \\
\hline María Paz & 48 & 1 & 25 & 23 & $52,08 \%$ & $47,92 \%$ \\
\hline Kennedy & 18 & 2 & 10 & 8 & $55,56 \%$ & $44,44 \%$ \\
\hline Colorados & 43 & $1-2$ & 25 & 18 & $58,14 \%$ & $41,86 \%$ \\
\hline $\begin{array}{l}\text { Colseguros } \\
\text { Norte }\end{array}$ & 18 & 3 & 12 & 6 & $66,67 \%$ & $33,33 \%$ \\
\hline Villa Alegría & 10 & $1-2$ & 8 & 2 & $80,00 \%$ & $20 \%$ \\
\hline
\end{tabular}

Fuente: autores

Tabla 6. Modelo multivariado de los principales factores asociados al reporte de episodios de diarrea en los últimos 6 meses.

\begin{tabular}{|c|c|c|c|c|c|c|}
\hline Episodios de diarrea & RP Crudo & $\mathbf{R P *}$ & \multicolumn{3}{|c|}{ IC $95 \%$} & $\boldsymbol{p}$ \\
\hline Número de síntomas que identifica el cuidador & $1,26(1,05$ a 1,51$)$ & 1,26 & 1,05 & a & 1,50 & 0,01 \\
\hline \multicolumn{7}{|l|}{ Aseguramiento } \\
\hline Contributivo/prepagada & & Referencia & & - & & - \\
\hline Subsidiado/SISBEN/No asegurado & $1,35(1,09$ a 1,69$)$ & 1,29 & 1,03 & a & 1,61 & 0,024 \\
\hline Edad del cuidador & $0,99(0,98$ a 1$)$ & 0,99 & 0,98 & a & 1,00 & 0,043 \\
\hline Conocimientos de diarrea transmitidos por familiar & $0,8(0,64$ a 1$)$ & 0,82 & 0,66 & a & 1,02 & 0,078 \\
\hline Preparado casero & $1,21(0,94$ a 1,57$)$ & 1,23 & 0,97 & a & 1,56 & 0,081 \\
\hline Descuajado & $1,32(1,06$ a 1,65$)$ & 1,21 & 0,96 & a & 1,51 & 0,102 \\
\hline Estrato 1 & & Referencia & & - & & - \\
\hline Estrato 2 & $0,92(0,71$ a 1,19$)$ & 0.92 & 0.71 & a & 1.19 & 0.51 \\
\hline Estrato 3 & $1,78(1,39$ a 2,27$)$ & 1,76 & 0,94 & a & 1,34 & 0,19 \\
\hline
\end{tabular}

*Cada Razón de prevalencia (RP) ajustada por las demás variables del modelo en la tabla.

Fuente: autores

La creencia popular más frecuente de causa de diarrea es la descuajadura; menos de la mitad de los cuidadores encuestados reconocen los alimentos y el agua contaminada como causa potencial de diarrea y menos de la tercera parte reconoce los virus, bacterias y parásitos como causa de esta enfermedad. Estudios en Nicaragua, muestran creencias populares de manera descriptiva, encontrando que al menos el $90 \%$ de las madres cuidadoras asociaban la diarrea con la nomenclatura mal de ojo y diarrea de movimiento (descuajadura) ${ }^{20}$. Teniendo en cuenta que la descuajadura dentro de este estudio fue la principal causa de EDA en niños menores de cinco años reportada por los cuidadores es importante ahondar más este tema desde perspectivas culturales para establecer estrategias educativas donde se articule la evidencia científica y conocimientos, actitudes y prácticas basadas en tradiciones.

Cervantes Baute, Bosch Govea y Armero Pérez (2001), exponen que los cuidadores creen como primer causa de diarrea algún tipo de parasito en un porcentaje de $42 \%$, mayor al encontrado en el presente estudio, la asociación de la diarrea con la contaminación de alimentos es expuesta por estos autores en menor 
porcentaje y no plantean la relación de causa con agua contaminada ${ }^{19}$. Otros investigadores como Alarcón, Aguirre, Álvarez encontraron que en Ica - Perú los cuidadores reconocen a los parásitos como principal causa de diarrea y con actitud de los cuidadores la suspensión de la lactancia materna ${ }^{20}$. Los signos de alarma para consulta inmediata a los servicios de salud más comúnmente identificados fueron la duración de la diarrea por más de 24 horas, fiebre de 39 grados centígrados o más y la intolerancia a la vía oral, a diferencia de los signos de deshidratación. Este desconocimiento del cuidador sobre signos de deshidratación se ha relacionado como un factor de riesgo en muerte por diarrea ${ }^{21,22,23}$. En otro estudio realizado por Lapeira, Acosta refiere que el 14,9\% reconoce los ojos hundidos como principal signo de alarma lo cual está relacionado de igual manera con un mayor riesgo de muerte.

En relación a prácticas del tratamiento el $88 \%$ de los cuidadores considera que hay que aumentar los líquidos y el $52 \%$ considera que hay que disminuir la cantidad de alimentos sólidos administrados durante el episodio de EDA. Datos consistentes con un estudio en República Dominicana donde el $61 \%$ de las madres desde el inicio de episodio de diarrea aumentan el aporte hídrico ${ }^{24}$. En cuanto a la utilización de SRO se encontró el $68,8 \%$ de los cuidadores conoce las SRO, pero solo un $49,4 \%$ las ha utilizado en el tratamiento de niños con diarrea, datos similares reportó Vásquez, en el 2014 cuyo estudio revela que un $51,2 \%$ usó SRO en el episodio de EDA ${ }^{25}$, otro estudio realizado en India en el 2011 evidencia que solo el 26\% sabía sobre el método correcto de preparación de $\mathrm{SRO}^{26}$.

En relación a los preparados caseros se encontró que los más frecuentemente utilizados para el manejo de la diarrea, en su orden son: el jugo de guayaba, el agua de manzanilla y el arroz tostado con un $25,60 \%$, $16,90 \%, 14,90 \%$ respectivamente. Datos similares arroja la investigación de Revollo y colaboradores en Bolivia, en 1995 y de Conde en Perú en $2015^{27}$.

La identificación de las características de la diarrea se asoció con un mayor reporte de episodios de diarrea en los últimos seis meses. Por tratarse de un estudio transversal, no se descarta la posibilidad de que sean precisamente los episodios de diarrea los que produzcan un efecto favorecedor para el reconocimiento de la duración de los síntomas, las características de la deposición y los signos de alarma, o bien que los cuidadores con bajo conocimiento sub reporten los episodios de diarrea de los niños a su cargo, debido a que no reconocen los síntomas o los signos de alarma.

Lo anterior podría explicar el gradiente encontrado entre mayor conocimiento de la diarrea y mayor reporte de los episodios, ya que es poco plausible postular que el conocimiento de los síntomas tiene un efecto favorecedor sobre la aparición de la diarrea en estos niños. Un estudio longitudinal está justificado y es pertinente para verificar esta hipótesis.

La edad del cuidador está relacionada a la consanguinidad con el niño, y es razonable pensar que una madre identifica más y mejor los síntomas diarreicos en sus hijos, y que esto podría explicar la asociación negativa entre edad del cuidador y la frecuencia de reporte de episodios; puesto que no se encontró asociación entre la edad o el parentesco y los conocimientos sobre diarrea, la explicación podría estar sustentada en una mayor intensidad del vínculo a nivel emocional, espacial y de tiempo derivada de la consanguinidad más proximal. No obstante, aunque se encontró asociación bivariada entre el mayor reporte de episodios de diarrea según si el cuidador era o no la madre, estadísticamente esta variable no cumplió criterios para ser candidata al modelo multivariado.

Los resultados del presente artículo provienen de un estudio transversal, por lo cual requieren ser interpretados y extrapolados de acuerdo al alcance de este tipo de estudio. El diseño transversal no permite validar hipótesis de causalidad, puesto que la relación temporal entre los factores asociados y la presentación de diarrea solo se puede establecer en un estudio longitudinal; sin embargo, las asociaciones estadísticas encontradas justifican y ameritan su inclusión en estudios posteriores de mayor nivel de evidencia. Asimismo, la exploración de prácticas consuetudinarias y creencias de la población aquí presentadas, evidencian la necesidad de análisis a profundidad complementarios con metodologías cualitativas.

\section{CONCLUSIONES}

En esta población prevalece el bajo nivel de conocimientos con respecto a las características, las causas, la gravedad, el manejo y la prevención de la diarrea aguda en más de la mitad de los cuidadores. Las creencias populares y las explicaciones de origen esotérico y sobrenatural se reportan con una alta 
prevalencia como causas de la enfermedad y los cuidadores de niños menores de cinco años de esta población de estrato bajo y medio bajo prefieren adquirir preparados comerciales de hidratación o un manejo casero en lugar del manejo con SRO. Las características del cuidador mostraron estadísticamente una asociación más fuerte y evidente que las circunstancias materiales sobre la prevalencia de periodo de diarrea aguda. Los resultados de este estudio sirven como base para justificar hipótesis para estudios longitudinales, tipo ensayo comunitario que permitan evaluar el efecto de intervenciones educativas sobre la presentación de diarrea aguda en niños de familias de medianos y escasos recursos.

\section{Comité de Ética}

En este estudio no se sometió a los individuos a ningún tratamiento experimental o algún tipo de examen invasivo, modificación conductual ni se indagó información sensible. El grupo de investigación conocía y se comprometió a cumplir con las pautas internacionales para la investigación en seres humanos, respetando durante todo su desarrollo lo establecido desde la declaración de Helsinki, el informe Belmont, las pautas de Consejo de Organizaciones Internacionales de las Ciencias Médicas (CIOMS) hasta la normativa nacional vigente en la resolución 008430 de 1993 del Ministerio de Salud de la República de Colombia. Acorde con la resolución 8430 de 1993 del Ministerio de Salud, se considera este proyecto como investigación sin riesgo (Artículo 11), dado su diseño, metodología y temática a indagar, por lo cual fue dispensado de consentimiento informado escrito (artículo 16, Parágrafo tercero). Se presentó ante el Comité de Ética de investigación de la UNAB obteniendo el aval del estudio.

\section{Financiamiento}

Los autores informan que la Universidad Autónoma de Bucaramanga, VII Convocatoria Interna de Proyectos de Investigación UNAB 2013-2014, es la institución que financio el presente estudio.

\section{Conflicto de InTerés}

Los autores manifiesta no haber tenido ningún conflicto de interés.

\section{Agradecimientos}

Los autores agradecen a la Universidad Autónoma de Bucaramanga, VII Convocatoria Interna de Proyectos de Investigación UNAB 2013-2014 y al Observatorio de Salud Pública de Santander, por el permanente apoyo y colaboración en la culminación del proceso de este estudio.

\section{Referencias Biblográficas}

1. Organización Mundial de la Salud. Nota descriptiva $\mathrm{N}^{\circ} 330$. Enfermedades diarreicas. [Internet]. 2013. Disponible en: http:// www.who.int/mediacentre/factsheets/fs330/es/

2. United Nations International Children's Emergency Fund. Progress for Children: A report card on water and sanitation (No. 5). Estados Unidos de América: UNICEF; 2006. Disponible en: https://www.unicef.org/publications/index_35977.html

3. Arguedas-Arguedas O. Vacunas contra Rotavirus. Acta Pediátr Costarric. 2009;20(2):88-91.

4. Díaz JJ, Echezuria L, Petit de Molero N, Cardozo MA, Arias A, Rísquez A. Diarrea aguda: Epidemiología, concepto, clasificación, clínica, diagnóstico, vacuna contra rotavirus. Arch Venez Puer Ped. 2014;77(1):29-40.

5. Black RE, Morris SS, Bryce J. Where and why are 10 million children dying every year? Lancet. 2003; 361(9376): 2226-34.

6. Organización Mundial de la Salud. Weekly Epidemiological Record. 2008;83(47):421-8.

7. Ministerio de la Protección Social. Instituto Nacional de Salud. Instituto Nacional de Vigilancia de Medicamentos y Alimentos. Plan de contingencia del sector salud para la prevención y control de cólera en Colombia. [Internet]. Bogotá: 2011. Disponible en: http://www.ins.gov.co/lineas-de-accion/Subdireccion-Vigilancia/ sivigila/Fichas\%20de\%20Notificacin \%20SIVIGILA/Plan\%20 contingencia\%20prevencion\%20y\%20control\%20colera.pdf

8. Así vamos en salud. Tasa de Mortalidad por Enfermedad Diarreica Aguda (EDA) en menores de 5 años - Georeferenciado. [Internet]. Bogotá: 2016. Disponible en: http://www.asivamosensalud. org/indicadores/estado-de-salud/tasa-de-mortalidad-porenfermedad-diarreica-aguda-eda-en-menores-de-5

9. Ramírez JC, Osorio H, Parra-Peña RI, Comisión Económica para América Latina y el Caribe. Escalafón de la competitividad de los departamentos de Colombia. [Internet]. Bogotá: 2007. Disponible en: http://repositorio.cepal.org/bitstream/ handle/11362/4815/S0700110_es.pdf?sequence $=1$

10. Otero JA, Uribe JC, Hormiga CM. Perfil de morbilidad y mortalidad en Santander. Revista del Observatorio de Salud Pública de Santander Colombia. 2013; 8(2):3-56.

11. Organización Mundial de la Salud. Investigaciones Operativas sobre Atención Integrada a las Enfermedades Prevalentes de la Infancia (AIEPI). [Internet]. Disponible en: http://www1.paho. org/Spanish/AD/DPC/CD/aiepi-27-inv-ops.htm

12. Fry S, Megan-Jane J, Faudrin-Flores P, Martínez-Moreno M. Ética en la práctica de enfermería, una guía para la toma de decisiones éticas. México, D.F: El Manual Moderno; 2010. p 46 - 47.

13. Fundación Salutia. Diarrea aguda una de las principales causas de muerte en los niños. Colombia. [Internet]. 2015. Disponible en: http://www.salutia.org/recreando_bienestar/index.php/13news/79-diarrea-aguda-una-de-las-principales-causas-demuerte-en-los-ninos

14. Gallardo-Lizarazo MP. Conductas, actitudes y prácticas de la madre o cuidador en el manejo de la enfermedad diarreica aguda en menores de cinco años. Rev Cienc Cuidad. 2015;12(2):39-56.

15. Gorter AC, Sánchez G, Pauw J, Pérez RM, Sandiford P, Smith GD. Childhood diarrhea in rural Nicaragua: beliefs and traditional health practices. Bol Oficina Sanit Panam. 1995;119(5):377-90.

16. Departamento administrativo Nacional de Estadística. Estratificación. [Internet]. Disponible en: https://www.dane.gov. co/files/geoestadistica/Preguntas_frecuentes_estratificacion.pdf 
17. Amare D, Dereje B, Kassie B, Tessema M, Mullu G, Alene B, et al. Maternal Knowledge and Practice Towards Diarrhea Management in Under Five Children In Fenote Selam Town, West Gojjam Zone, Amhara Regional State, Northwest Ethiopia. J Infect Dis Ther. 2014:2:182.

18. Caruso B, Stephenson R, Leon J. Maternal behavior and experience, care access and agency as determinants of child diarrhea in Bolivia. Rev Panam Salud Publica. 2010;28(6):429-39.

19. Cervantes-Baute IC, Bosch-Govea M, Armero-Pérez G. Valoración del conocimiento de las madres sobre las diarreas y su prevención. Rev Cubana Enfermer. 2001;17(1)56-9.

20. Jhonnel Alarco J, Aguirre-Cuadros E, Álvarez-Andrade EV. Conocimiento de las madres sobre la diarrea y su prevención en un asentamiento humano de la provincia de Ica, Perú. Rev Clín Med Fam. 2013:6(1):25-31.

21. Delgado MF, Sierra CH, Calvache JA, Ríos AM, Mosquera C, Salas I, Agredo F, Meneses R. Conocimientos maternos sobre signos de peligro en diarrea aguda en el marco de la estrategia AIEPI. Colomb Med. 2006;37:293-98.

22. Terra de Souza AC, Peterson KE, Andrade FM, Gardner
J, Ascherio A. Circumstances of post-neonatal deaths in Ceara, Northeast Brazil: mothers' health care-seeking behaviors during their infants' fatal illness. Soc Sci Med. 2000;51(11):1675-93.

23. Trostle JA, Yepez-Montufar JA, Ríos AM, Mosquera-Salas C, Meneses R. Males diarreicos en la costa ecuatoriana: cambios socioambientales y concepciones de salud. Cad. Saúde Pública. 2010;26(7):1334-44.

24. Vásquez L, Herrera D, Segura M. Conocimientos sobre hidratación oral por las madres de pacientes de 1 a 5 años de vida con enfermedad diarreica aguda. Horiz Med. 2014;14(3):24-7.

25. Ahmad S, Khalique N, Khan IM, Ansari MA, Khan Z. Do the mothers in rural Aligarh know about home based management of acute diarrhea. Biology and Medicine. 2011;3(2):76-80.

26. Revollo M, Rita M, Aguilar L, Zamora AM. Manejo de la diarrea en el hogar, estudio en la ciudad de El Alto. Rev. Soc. Boliv. Pediatr. 1995;34(2):43-50.

27. Conde-Chavez LK. Prácticas y el resultado en el manejo inicial de la diarrea aguda en niños menores de 5 años por madres peruanas. Análisis ENDES. 2013 [Tesis]. Perú: Universidad Nacional Mayor de San Marcos. Facultad de Medicina; 2015. 